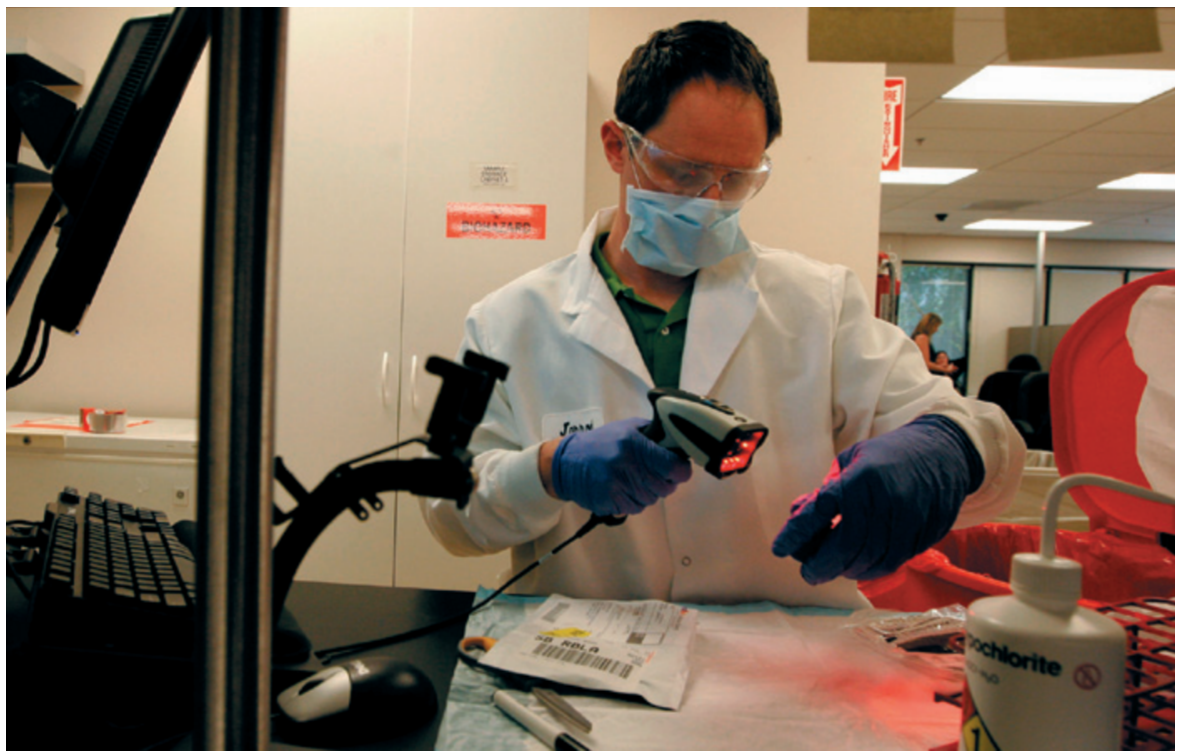

A lab technician scans a consumer gene-testing kit.

\title{
The FDA is overcautious on consumer genomics
}

\author{
A US drug-agency clampdown is unwarranted \\ without evidence of harm, say Robert C. Green \\ and Nita A. Farahany.
}

$\mathrm{S}$ ince 2007, people have been able to spit in a tube, ship it to a company and later log on to a website to learn what their DNA reveals about non-medical traits such as hair texture and ancestry, as well as whether they carry DNA variants associated with increased risks for diseases including type 2 diabetes and Alzheimer's. More than 500,000 people have bought such tests.

On 22 November last year, the company that has performed the bulk of these tests received a letter from the US Food and Drug Administration (FDA). The FDA ordered 23 andMe, based in Mountain View, California, to "immediately discontinue marketing" its testing kit and personal genome services, which the agency says offer medical advice and so require regulatory approval. "Serious concerns are raised if test results are not adequately understood by patients or if incorrect test results are reported," the FDA wrote. Notably, it said, genetic results could drive consumers to take extreme steps, such as having unnecessary surgery to prevent cancer. Consumers might also abandon or alter prescribed treatments without consulting health professionals, the letter alleged.

Two weeks after receiving the letter, 23andMe took steps to allay the FDA's concerns. It continues to offer DNA testing and analysis, but no longer provides new consumers with genetic interpretations that relate to health.

The FDA invoked the precautionary principle - acting on the basis of speculations of potential harm rather than reported harm. Although the FDA requires manufacturers of drugs and diagnostic devices to prove safety and efficacy before marketing, we feel that this approach is unwarranted in regulating 23andMe's personal genome service.

In its earlier warning letters, the agency said that genomic health reporting "appears to meet the definition" of a medical device. In its November correspondence, it states that 23andMe's service is a medical device. The FDA now claims jurisdiction over companies that provide health-related interpretations with genomic data (but not those that provide genomic data alone). But such interpretations, particularly about common genetic variants, relate only indirectly to preventing or diagnosing disease. In this sense, it is like the inferences drawn about rapid weight loss measured by a bathroom scale.

The FDA cannot reasonably regulate all such indirect information as medical devices. Moreover, as the court cases of Sorrell v. IMS Health (2011) and United States v. Caronia (2012) demonstrate, doing so could put FDA regulations in greater tension with the First Amendment of the US Constitution, which protects the rights of individuals to receive information, and of 'commercial speech'1. Given this backdrop, the agency should avoid restricting consumer genomic testing unless faced with empirical evidence of harm.

Certainly, there are legitimate concerns about 23andMe's approach. Although the accuracy of the technology used is considered to be high, there are no agreed standards to which the company can conform for validating hundreds of simultaneous variant calls. There is also controversy about how to evaluate the accuracy of risks estimated using multiple variants or across ethnicities ${ }^{2,3}$. And consumers might not read or fully understand the company's clear statements that its tests identify only the most common genetic variants and cannot substitute for genetic testing ordered by physicians to assess specific indications, such as a family history of cancer.

Nonetheless, as scholars who study how individuals respond to their own genetic information, we contend that the FDA's precautionary approach may pose a greater threat to consumer health than the harms that it seeks to prevent. Data from more than 5,000 participants suggest that consumer genomics does not provoke distress or inappropriate treatment.

\section{EMERGING EVIDENCE}

Over the past five years, we and others have surveyed people who have received consumer genomics results, asking whether they understood them and whether the findings provoked distress, prompted a visit to a doctor or triggered a change in medication or lifestyle.

In 2009, the Scripps Genomic Health Initiative (SGHI) recruited more than 3,000 individuals from health and technology companies and offered subsidized testing through Navigenics (a company that is now owned by Life Technologies and no longer offers consumer testing). Surveys were administered before the participants received results, and 3 and 12 months afterwards. Responses from more than 2,000 participants showed no measurable changes in anxiety or psychological health ${ }^{4,5}$.

Consumers who accept subsidized testing might be less anxious than those who seek it out, but we found similar responses in the Impact of Personal Genomics (PGen) Study funded by the US National Institutes of Health (NIH) and jointly led by R.C.G. and health-behaviour researcher Scott Roberts. In 2012-13, we surveyed more than 1,800 customers from two consumer genomics companies - Pathway Genomics and 23andMe. (Pathway Genomics has subsequently changed its business model to focus on tests ordered by physicians.) Preliminary data suggest that on average, customers were briefly 
less anxious than their baseline after receiving their results, and never showed elevated anxiety or distress over the following year. These findings were consistent with those observed in the REVEAL (Risk Evaluation and Education for Alzheimer's Disease) Study, a series of NIH-funded randomized trials carried out between 2000 and 2013. More than 700 volunteers, most of whose family members had been diagnosed with Alzheimer's disease, underwent genetic tests assessing their own risk of this disease, and roughly $40 \%$ learned that they have increased risk. But even this potentially frightening disclosure caused only modest and transient distress ${ }^{6,7}$.

A 2010 survey by researchers at Johns Hopkins University in Baltimore, Maryland, of more than 1,000 customers of three companies found that just over one-quarter of people share their results with physicians in the first few months after receiving them. Similar findings were obtained by the SGHI and PGen Study ${ }^{5,8}$. The Hopkins and PGen studies also found that fewer than $1 \%$ of customers reported altering any prescription medicines on the basis of their results without first consulting a physician (see 'Taking action').

Just as patients sometimes misunderstand physicians during office visits, customers sometimes misconstrue results provided by consumer genomics companies. In the Hopkins survey, participants were asked to interpret hypothetical results. Between $5 \%$ and 9\% interpreted straightforward messages incorrectly - stating that results showing increased risk instead indicated decreased or equal risk, or vice versa.

The FDA is particularly concerned about how people might respond to learning that they have $B R C A$ gene mutations that increase their risk of breast and ovarian cancers. Early evidence suggests that consumers respond appropriately. In a study carried out by 23 andMe, the company sent interview invitations to 136 customers who carried pathogenic mutations ${ }^{9}$. Of the 32 who accepted, 14 men and 11 women had learned for the first time through consumer testing that they carried a high-risk $B R C A$ mutation. All of the women had consulted health-care professionals with their results, and all but one (who elected for surveillance and not surgery) had their tests repeated. Their behaviour after learning this information was no different from that of people who discover these mutations through medical channels.

These interviews also revealed that 30 family members of those carrying a pathogenic mutation decided to get tested themselves; 13 of them tested positive for the high-risk mutation and so received potentially life-saving information they might not have obtained otherwise.

Clearly this is a very small study and conducted by an interested party. It provides no information about the majority of $B R C A$-positive customers, who were not interviewed. Nor did the study evaluate whether women with undetected mutations and a strong family history of breast cancer might have been falsely reassured (despite clear company messages that their BRCA testing is not comprehensive) and, as a result, mistakenly elected not to pursue testing in a medical context. Obtaining a truly representative sample is extremely difficult because consumers who participate in surveys might differ from those who do not, and each of the surveys suggest that early customers of genomics services are wealthier and better educated than the general population, and more likely to be white.

More systematic research is needed to assess the outcomes of consumer genomics testing. The PGen Study results will be available by the end of this year, and further surveys could expand this work to a much larger sample, performing standardized follow-ups on consumers who receive certain high-impact results. However, if consumer genomics is halted, researchers will not be able to continue gathering data to better assess what the benefits and harms could be.

\section{DEMOCRATIZING HEALTH CARE}

We find the FDA's precautionary approach to 23 andMe particularly troubling because

\section{TAKING ACTION}

After receiving genomics results, $42 \%$ of 1,051 surveyed people reported positive

changes in their health behaviour. Only $1 \%$

of all respondents altered a prescription

treatment without consulting a doctor.
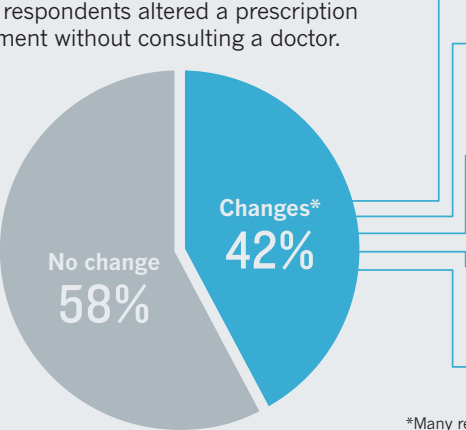

Dietary patterns

$72 \%$

Exercise habits

$61 \%$

Supplements

$170 /$ with medical $210 /$ without medical

$17 \%$ consultation $21 \%$ consultation

Non-prescription drugs

$10 \%$ with medical $70 /$ without medical

Prescription drugs

$110 /$ with medical $20 /$ without medical

*Many respondents reported more than one change, so percentages total more than $100 \%$. it could presage similar actions against other consumer health products. In its recent guidance on mobile health applications, the FDA left open the possibility that it will regulate as medical devices information-based products such as questionnaires that evaluate the risk of a heart attack or the plethora of fitness trackers that help people to follow their weight, body temperature, heart rate, sleep patterns and more. Many operate as standalone or companion software for predicting risks including the likelihood of sleep disorders, seizures or heart attacks. Downloads and installations of these applications are expected to grow from 156 million in 2012 to 248 million in 2017 (ref. 10).

Such consumer products could democratize health care by enabling individuals to make choices that maximize their own health. They follow the historical trend of patient empowerment that brought informed-consent laws, access to medical records and now direct access to electronic personal health data.

We believe that 23 andMe should be more transparent about how accurate its genotyping chips are, and even more forthcoming about the limitations of its computer algorithms used for estimations of risk. But regulatory constraints might stifle consumer genomics and other emerging products that could make society healthier and that do not fit neatly into the model of physiciandriven health care. The effects of these products should be monitored but, as long as emerging empirical data show no evidence of harm, we urge the FDA to let consumer genomics testing proceed.

Robert C. Green is in the Division of Genetics, Brigham and Women's Hospital and Harvard Medical School, and the Partners HealthCare Center for Personalized Genetic Medicine, Boston, Massachusetts. Nita A. Farahany is at the Duke University School of Law and the Duke Institute for Genome Sciences and Policy, Durham, North Carolina. e-mails: rcgreen@genetics.med.harvard.edu; farahany@duke.edu

1. Carver, K. H. Food Drug Law J. 63, 151-215 (2008).

2. Kalf, R. R. J. et al. Genet. Med. http://dx.doi. org/10.1038/gim.2013.80 (2013)

3. Swan, M. Genet. Med. 12, 279-288 (2010)

4. Bloss, C. S. et al. Genet. Med. 12, 556-566 (2010)

5. Bloss, C. S., Schork, N. J. \& Topol, E. J. N. Engl. J. Med. 364, 524-534 (2011).

6. Green, R. C. et al. N. Engl. J. Med. 361, 245-254 (2009).

7. Lautenbach, D. M., Christensen, K. D., Sparks, J. A. \& Green, R. C. Annu. Rev. Genomics Hum. Genet. 14, 491-513 (2013).

8. Kaufman, D. J., Bollinger, J. M., Dvoskin, R. L. \& Scott, J. A. J. Genet. Couns. 21, 413-422 (2012).

9. Francke, U. et al. PeerJ 1, e8 (2013).

10. Topol, E. The Creative Destruction of Medicine (Basic, 2012).

R.C.G. declares competing interests: see go.nature. com/qpucpq for details. 